$\mathbb{T}$ periodica polytechnica

\author{
Transportation Engineering \\ $39 / 2(2011) 91,97$ \\ doi: $10.3311 /$ pp.tr.2011-2.08 \\ web: http://www.pp.bme.hu/tr \\ (C) Periodica Polytechnica 2011
}

RESEARCH ARTICLE

\section{Driveline torque observer for heavy duty vehicle}

Balázs Trencséni / László Palkovics

Received 2010-09-07

\begin{abstract}
In addition to fuel consumption and efficiency the expectations in driving comfort of heavy duty vehicles were substantially increased in the last years. The driveline has an essential role in drivability performance of the vehicle. Since it determines the driving efficiency as well, simple, but robust devices were quite widespread in production, but these can usually make driving more difficult to the driver. This means there is a hard trade-off between efficiency and driving comfort. Application of control logics can help to find the balance between simplification of hardware components and their operation. Enhancing the drivability without any inefficient additional assembly leads to higher level of safety since it makes driver's job easier, and so it can further increase the efficiency of transportation.
\end{abstract}

\section{Keywords}

heavy duty vehicle $\cdot$ driveline $\cdot$ modal analysis $\cdot$ observer design

\section{Balázs Trencséni}

Department of Automobiles, BME, H-1111 Budapest, Stoczek u. 6, Hungary e-mail: trencseni@auto.bme.hu

\section{László Palkovics}

Department of Automobiles, BME, H-1111 Budapest, Stoczek u. 6, Hungary e-mail: palko@auto.bme.hu

\section{Introduction}

Driveline control has attracted considerable attention recently in the automotive industry. There are many possible applications of driveline control that could benefit vehicle behavior, such as reduction of oscillations, improvement of gearshift quality and in general the drivability, but they have also profitable aspects to fuel economy and environmental impact. Since the components of the driveline are elastic, mechanical resonances may occur. Oscillations in the driveline may be excited first of all during transients of input torques for instance at gear-shifting or changing accelerator pedal position rapidly.

The problem of estimating the state variables of a dynamic system for given observations of the output variables is of fundamental importance in control theory since many feedback control designs require availability of the states of the plant that has to be controlled. Automotive powertrains are furthermore often affected by nonlinearities. A rather accurate estimation of shaft torque is needed for successful control of vehicle powertrains. However, shaft torque is difficult and expensive to measure directly. In this paper a driveline torque observer will be discussed. This includes examination to suitable system design and Kalman filter theory. The Kalman filter is an observer that contains a system model. This system model has to be sufficiently accurate but not very complex.

\section{Generation of suitable model}

The driveline model, which contains each driveline elements separately according to the construction of a $4 \times 2$ truck, has at least 8 degrees of freedom [5]. It was already analyzed to be suitable for observer design [5], but it is rather complex to realtime control applications. Using modal analysis sufficient model structure and reduced degree of freedom of the model can be deduced. This procedure agrees the determination of natural frequencies and the oscillation forms; the eigenvectors belong to given frequencies. Knowing the eigenvector of relevant natural frequencies the importance of different flexible elements in the driveline may be established in the given frequency range.

First of all, the complex eight-mass driveline model described by motion equations [5] was linearized and its mass, stiffness 
and damping matrices were noted. The motion equations of each degree of freedom (inertias) of a rotational system can be written summarized in general matrix form:

$$
\mathbf{M} \ddot{\mathbf{q}}+\mathbf{C} \dot{\mathbf{q}}+\mathbf{K q}=\mathbf{f}(t),
$$

where $\mathbf{M}, \mathbf{K}$ and $\mathbf{C}$ sign mass, stiffness and damping matrices and $\mathbf{q}$ vector contains the general coordinates of motion, in which $\theta$ denotes always angle position of different powertrain elements [5]. If the $M$ marks torque, $F_{\text {traction }}$ is force on the wheel-road contact patch and $r_{w}$ marks wheel radius, then it can be written:

$$
\begin{gathered}
\mathbf{q}=\left[\begin{array}{llllllll}
\theta_{m} & \theta_{\text {clutch }} & \theta_{t r 1} & \theta_{t r 2} & \theta_{k k 2} & \theta_{\text {bevel }} & \theta_{w} & \theta_{\text {tire }}
\end{array}\right]^{T}, \\
\mathbf{f}=\left[\begin{array}{cccccccc}
M_{m} & 0 & -M_{\text {retarder }} & 0 & 0 & 0 & -M_{\text {brake }} & -F_{\text {traction }} r_{w}
\end{array}\right]^{T} .
\end{gathered}
$$

For model simplification the nonlinearities were neglected; clutch characteristic was changed to a linear one, backlashes were not taken into consideration and the total vehicle rolling mass was coupled to the tire as a large moment of inertia.

The determination of the natural frequencies of the system is in fact an eigenvalue problem, in that the excitation of the system is zero, i.e. $\mathbf{f}(t)=0$. The dynamic system described $\dot{\mathbf{x}}=\mathbf{A x}$ according to definition of eigenvalue problem has got $\lambda$ eigenvalue and $\mathbf{v}$ eigenvector, which satisfy the following equation:

$$
\mathbf{A v}=\lambda \mathbf{v}
$$

In this case the general motion equation can be ordered as follows $\mathbf{f}(t)=0$ :

$$
\ddot{\mathbf{q}}=-\mathbf{M}^{-1} \mathbf{K q}-\mathbf{M}^{-1} \mathbf{C} \dot{\mathbf{q}} .
$$

If choose $\mathbf{x}=\left[\begin{array}{c}\mathbf{q} \\ \dot{\mathbf{q}}\end{array}\right]$, then $\mathbf{A}$ dynamic matrix will have the form:

$$
\mathbf{A}=\left[\begin{array}{ll}
\mathbf{0} & \mathbf{I} \\
-\mathbf{M}^{-1} \mathbf{K} & -\mathbf{M}^{-1} \mathbf{C}
\end{array}\right]
$$

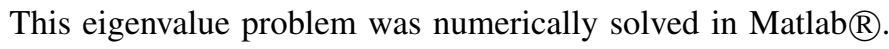
The system has 8 degrees of freedom and 16 states. The analysis executed for each transmission gear ratio delivers 16 natural frequencies and eigenvectors concerned. Two of 16 eigenvalues are zeros, these describe the rigid body motion of the system. The other 14 eigenvalues are conjugate complex and describe the natural oscillations of the system. Natural frequencies of the system parameterized for a given $4 \times 2$ truck are shown in Table 1 $[\mathrm{Hz}]$.

As can be seen in Table 1 , in the really comfort relevant low frequency range only the first, possibly the second natural oscillation mode are to be found. The eigenvector belongs to the significant first oscillation mode is shown in Fig. 1. The amplitudes were reduced to the engine concerning gear ratios of transmission and final gear. This figure shows us that especially at lower gears the rotational masses from engine to the rear axle oscillate with the same amplitude, while wheel and vehicle body swing with opposite amplitude. At higher gears the amplitudes of wheel and vehicle body will be smaller, while amplitudes of masses from transmission and rear axle show slight losses compared with the amplitude of the engine, but the masses on the front part of the driveline move rather in same phase. On the other hand the wheel oscillates in counter phase to engine at lower gear, then in phase at higher gear.

The result of modal analysis shows consequently, that first of all driveshaft has got a high importance according to dynamic behavior of the driveline. The second most significant is tire elasticity, and probably the propeller shaft follows it. But the engine and transmission can be treated as one single unit, i.e. the elements from engine to transmission output can be substituted by one moment of inertia. In this sense a two-mass, i.e. $2^{\text {nd }}$ order model is seemed to be sufficient to return the motion of the system in the comfort-relevant lower frequency range and at lower gears.

The reduced model means smaller computing demand. Therefore the observer design will be based on a simple twomass rotational model. Focusing on the nonlinearities it can be declared that the tire slip is the same as damping concerning its effect; therefore it can be added to tire damping. If the same modal analysis is executed with the lower stiffness value of the nonlinear clutch characteristic, then the increasing significance of clutch elasticity at higher gears will be demonstrated. But at lower gears, where the driving comfort can be destroyed significantly by driveline oscillations, the following statements remain valid; the lower clutch stiffness indicates approximately same importance as propeller shaft, while driveshaft and tire represent main elastic elements in the driveline. Furthermore, it should be noted that this reduced stiffness is only valid in a small torsion range, which fact reduces the importance of clutch even further. That is the reason why clutch stiffness was ignored during observer design.

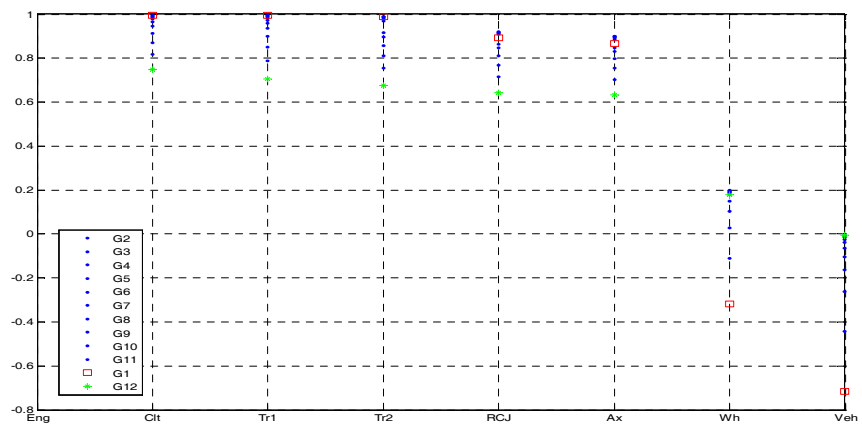

Fig. 1. The first mode eigenvector of the driveline model for each gears of test vehicle

In accordance with the two statements above, between inertias of the driveline, of which speed are measurable, namely between the engine-transmission unit and wheel, the only nonlinearities are the backlashes in gears and joints that can be summarized into one backlash. Look at the measured torque in the driveline of the vehicle equipped with torque sensor the effect of backlash can be observed. That was the motivation to design 
Tab. 1. Natural frequencies $[\mathrm{Hz}]$ in each gear

\begin{tabular}{|c|c|c|c|c|c|c|c|c|c|c|c|c|c|}
\hline & \multicolumn{13}{|c|}{ Gear } \\
\hline \multirow{6}{*}{$\begin{array}{l}\frac{0}{0} \\
\frac{0}{2}\end{array}$} & $\ldots$ & 1 & 2 & 3 & 4 & 5 & 6 & 7 & 8 & 9 & 10 & 11 & 12 \\
\hline & 5 & 379.2 & 366.9 & 377.5 & 365.6 & 374.9 & 363.9 & 207.9 & 208.8 & 209.3 & 211.7 & 226.8 & 232.9 \\
\hline & 4 & 113.8 & 113.8 & 115 & 115 & 117.8 & 117.9 & 103.8 & 103.3 & 117.5 & 118 & 135.2 & 136.6 \\
\hline & 3 & 65.35 & 55.06 & 63.77 & 53.71 & 60.52 & 50.95 & 54.04 & 46.45 & 46.03 & 40.04 & 38.58 & 34.33 \\
\hline & 2 & 17.88 & 17.88 & 17.88 & 17.87 & 17.86 & 17.84 & 17.71 & 17.67 & 17.59 & 17.49 & 17.36 & 17.14 \\
\hline & 1 & 0.77 & 0.9 & 1.09 & 1.33 & 1.63 & 2.02 & 2.53 & 3.15 & 3.94 & 4.81 & 5.71 & 6.73 \\
\hline
\end{tabular}

a nonlinear observer which is able to treat the nonlinearities of backlash in the driveline and makes the torque estimation more accurate even if the engine goes from braking to acceleration or vice versa. The observer bases on the simplified model that is shown in Fig. 2

The moments of inertia of elements from engine to final gear were summarized into $J_{\text {EngTrDiff }}$. Here will be presented the two masses model, in which the moments of inertia of wheel, tire and reduced vehicle rolling mass were denoted with $J_{w}+$ $J_{\text {tire }}+m_{\text {roll }} r_{w}^{2}$. The elastic elements were combined into $k_{\text {drivel }}$ resultant stiffness and $c_{\text {drivel }}$ resultant damping. The $b$ viscous damping coefficients for gear elements can be further applied to considering losses, $i_{t r}$ and $i_{D}$ denote the gear ratios in gearbox and in final gear respectively.

Above all the motion equations of the simplified driveline model were written if backlash is engaged (or zero):

$J_{\text {EngTrDiff }} \cdot \ddot{\theta}_{m}=$

$u-\frac{k_{\text {drivel }}}{i_{t r} i_{D}}\left(\frac{\theta_{m}}{i_{t r} i_{D}}-\theta_{w}\right)-\frac{c_{d r i v e l}}{i_{t r} i_{D}}\left(\frac{\dot{\theta}_{m}}{i_{t r} i_{D}}-\dot{\theta}_{w}\right)-b_{\text {total }} \dot{\theta}_{m}$

$\left(J_{w}+J_{\text {tire }}+m_{\text {roll }} r_{w}^{2}\right) \cdot \ddot{\theta}_{w}=$

$k_{\text {drivel }}\left(\frac{\theta_{m}}{i_{t r} i_{D}}-\theta_{w}\right)+c_{\text {drivel }}\left(\frac{\dot{\theta}_{m}}{i_{t r} i_{D}}-\dot{\theta}_{w}\right)-\frac{1}{2} A_{w} c_{w} \rho r_{w}^{3} \dot{\theta}_{w}$

$-m_{\text {roll }} g c_{w r 2}\left(1+0.06 r_{w}^{2} \dot{\theta}_{w}\right) r_{w}-m_{\text {roll }} g r_{w} \cdot \delta$,

where drive resistances were linearized omitting the quadratic functions in equations of air drag and rolling resistance; and assumed that $\cos \delta \approx 1$ and $\sin \delta \approx \delta$ at small $\delta$ road incline angles.

The drive resistance was implemented into the observer according to Eq. (2). However, since the road slope angle $\delta$ is unknown, it has to be estimated also. This was done by introducing the road slope as a new state of the system. Since the road slope changes slowly compared to torque excitation of the driveline, its derivative was considered to be constant but disturbed with (white) noise. This way of resistance modeling makes it possible that braking on wheel is interpreted as a resistance as well.

Introducing the state vector

$$
\mathbf{x}=\left[\begin{array}{lllll}
\dot{\theta}_{m} & \dot{\theta}_{w} & \Delta \theta & \delta & v_{\delta}
\end{array}\right]^{T},
$$

where $\Delta \theta$ denotes the torsion of driveline elasticity:

$$
\Delta \theta=\frac{\theta_{m}}{i_{t r} i_{D}}-\theta_{w},
$$

and $v_{\delta}$ denotes derivatives of road inclination angle, the equations of system can be written in matrix form.

\section{Extended Kalman filter}

Looking at the backlash it can be seen that it causes zero torque transmission in driveline during its traverse, i.e. it means torque break. That is the reason why backlash is a major source of drivability limitations. Linearization of backlash can not be a suitable method to manage it; this would efface exactly its nature. The non-continuous backlash characteristic can be approached with only high ordered polynomials, which on the one hand have expected accuracy only inside a small working range and cause huge discrepancy out of their scope, and on the other hand they make the computing of partial derivatives for Liebracket necessary to feedback linearization extremely hard. At the same time the rotating mechanical system affected by backlash can not be written in affine form, because varying parameters depend directly on a state of the system, in this case on the angle difference between the two sides on the backlash.

According to the considerations over linearization and gain scheduling, it was investigated in a method, which involves linear models to cover the whole operating range, but it does not demand to solve a Riccati equation on-line. This leads to an observer the same as the extended Kalman filter, but simplified in gain variance. (That is the so-called constant gain extended Kalman filter.) Instead of gain calculation in each time step predefined feedback gains for certain linearization points are switched depending on the state vector and its derivative. In addition to the continuous state variables such a model contains at least two discrete states that determine which gain is actually switched, i.e. the observer may be considered a hybrid system. To achieve the goal a new backlash model was introduced.

The so-called physical backlash model was introduced in [4]. This model makes it possible to treat the driveline in two states: if backlash is actually engaged, then driveline is in contact mode, or if backlash is actually traversing, then driveline is in backlash mode. It introduces the relative angle difference between the two sides of backlash as a state variable, which is strictly speaking equal to the position inside the backlash. The 


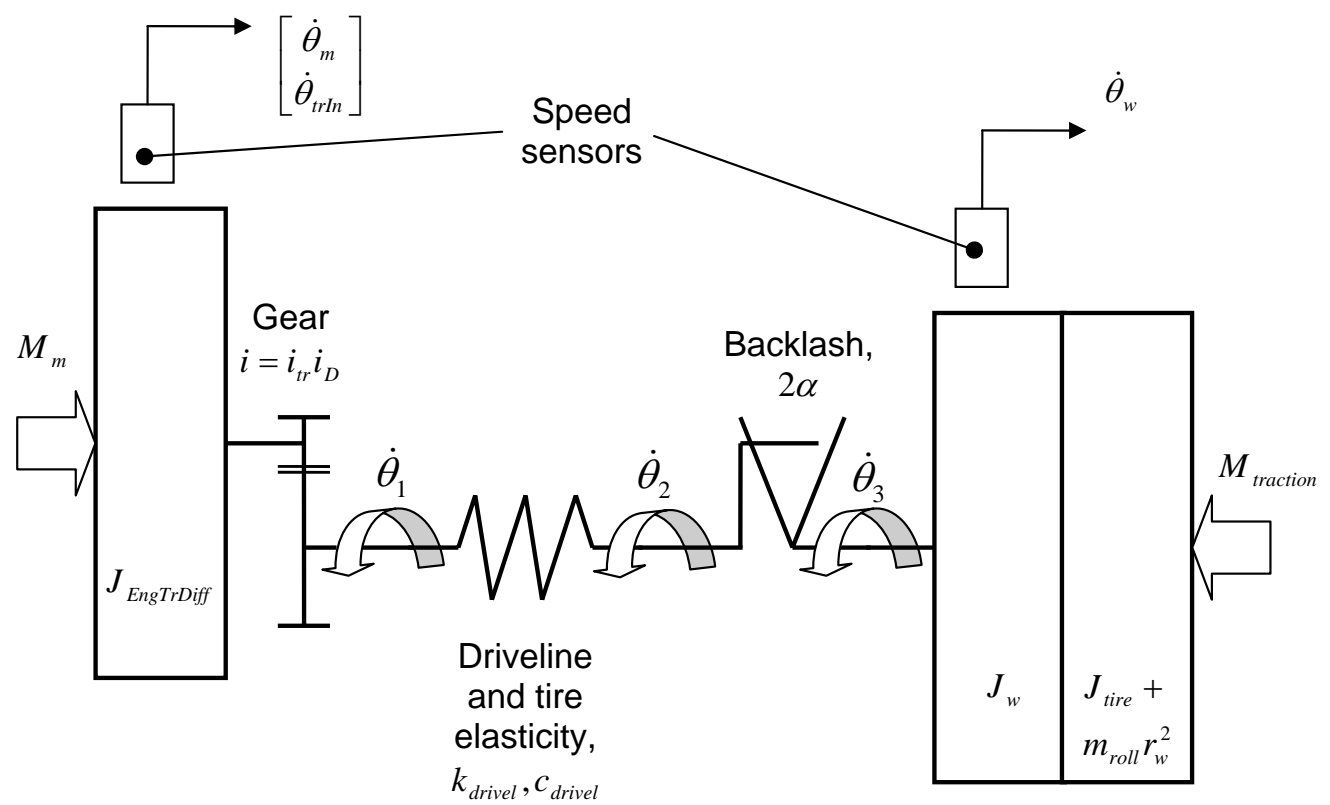

Fig. 2. Simplified driveline model for observer design

switch between contact and backlash modes is based on the position state variable and its derivative. The applied classical dead-zone model is shown to be wrong when the shaft has nonnegligible internal damping, because it gives the output shaft torque as a function of displacement between the input and output elements only. This new more exact backlash model takes the internal damping also into account. The shaft torque is given by:

$M_{\text {driveline }}=k_{\text {drivel }}\left(\Delta \theta-\theta_{b}\right)+c_{\text {drivel }}\left(\frac{\dot{\theta}_{m}}{i_{\text {tr }} i_{D}}-\dot{\theta}_{w}-\dot{\theta}_{b}\right)$.

where $\Delta \theta$ is the total shaft displacement as defined before, $\dot{\theta}_{m}$ and $\dot{\theta}_{w}$ stand for the speeds of engine as drive element and of wheel as driven element and $\theta_{b}$ denotes the position in the backlash: $\theta_{b}=\theta_{2}-\theta_{3}$, where $\theta_{2}$ is the angle of driving shaft at the backlash according to marking in Fig. 2 and $\theta_{3}=\theta_{w}$ in our discussion.

With $\alpha$ denoting half backlash size, the backlash position is governed by the following dynamics:

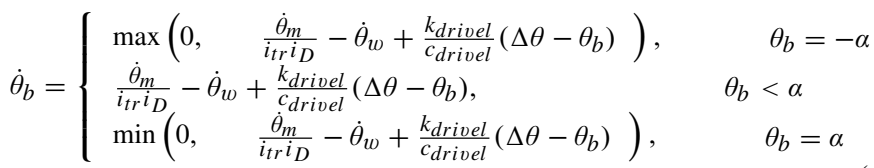

We have now a nonlinear dynamical system, which is suitable to augment the two-mass model with a new state of backlash position $\theta_{b}$.

Next the exploiting of the structure of the physical backlash model will be shown, how the driveline model can be written as a system switching between two linear models called - as mentioned above - backlash mode and contact mode.

Using updated state vector:

$$
\mathbf{x}=\left[\begin{array}{llllll}
\dot{\theta}_{m} & \dot{\theta}_{w} & \Delta \theta & \theta_{b} & \delta & v_{\delta}
\end{array}\right]^{T}
$$

The backlash dynamics based on Eq. (4) can be rewritten as:

$$
\begin{aligned}
& \dot{\theta}_{b}=\dot{x}_{4}=H(\mathbf{x})= \\
& \left\{\begin{array}{ccc}
\max (0, & h(\mathbf{x})) & x_{4}=-\alpha \\
h(\mathbf{x}), & & \left|x_{4}\right|<\alpha \\
\min (0, & h(\mathbf{x})) & x_{4}=\alpha
\end{array}\right.
\end{aligned}
$$

where $h(x)$ is linear:

$$
h(\mathbf{x})=\mathbf{a}_{\text {bldyn }} \mathbf{x}=\left[\begin{array}{llllll}
\frac{1}{i_{t r} i_{D}} & -1 & \frac{k_{\text {drivel }}}{c_{\text {drivel }}} & -\frac{k_{\text {drivel }}}{c_{\text {drivel }}} & 0 & 0
\end{array}\right] \mathbf{x .}
$$

The linearization of the nonlinear process model to derive the extended Kalman filter is in this case equal to linearization of the $H(\mathbf{x})$ function.

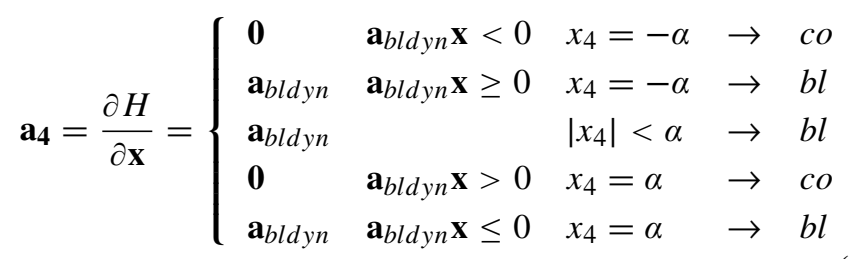

So the nonlinearity consists of only two distinct modes, both linear. The fourth row of dynamic matrix $\mathbf{A}$, denoted $\mathbf{a}_{4}$ takes the value $\mathbf{a}_{b l d y n}$ or zero row vector value, depending on the five sets of conditions in Eq. (??). The $\mathbf{a}_{\text {bldyn }}$ row vector corresponds to the disengaged driveline, if backlash is being open, or in contact, but moving toward opening. The value of zero row vector corresponds to permanent stable contact.

Substitute the torque written in Eq. (3) into motion equations Eq. (??) - (??) and apply the updated state vector $\mathbf{x}$, the state-space representation turns into following matrices in con- 
tact mode:

$\mathbf{A}_{c o}=\left[\begin{array}{ll}-\frac{c_{\text {drivel }} /\left(i_{\text {tr }}^{2} i_{D}^{2}\right)+b_{\text {total }}}{J_{\text {EngTrDiff }}} & \frac{c_{\text {drivel }} /\left(i_{\text {tr }} i_{D}\right)}{J_{\text {EngTrDiff }}} \\ \frac{c_{\text {drivel }} /\left(i_{\text {tr }} i_{D}\right)}{J_{w}+J_{\text {tire }}+m_{\text {roll }} r_{w}^{2}} & \frac{-c_{\text {drivel }}-\frac{1}{2} A_{w} c_{w} \rho r_{w}^{3}-m_{\text {roll }} g c_{w r 2} 0,0036 r_{w}^{3}}{J_{w}+J_{\text {tire }}+m_{\text {roll }} r_{w}^{2}} \\ \frac{1}{i_{\text {tr }} i_{D}} & -1 \\ 0 & 0 \\ 0 & 0 \\ 0 & 0\end{array}\right.$

As mentioned before, during backlash mode no torque can be transmitted that leads to disassembling of driveline, which is treated by the backlash model introduced above. In backlash mode, the $J_{\text {EngTrDiff }}$ front part of the driveline rotates independently on $J_{w}+J_{\text {tire }}+m_{\text {roll }} r_{w}^{2}$ (rear part of the driveline), excited by $M_{m}$ engine torque and $M_{\text {traction }}$ traction (or load) torque correspondingly (see Fig. 2). This fact causes very simple equations of motion for both moments of inertia, namely:

$$
\begin{aligned}
& J_{\text {EngTrDiff }} \cdot \ddot{\theta}_{m}=u-b_{\text {total }} \dot{\theta}_{m} \\
& \left(J_{w}+J_{\text {tire }}+m_{\text {roll }} r_{w}^{2}\right) \cdot \ddot{\theta}_{w}=-\frac{1}{2} A_{w} c_{w} \rho r_{w}^{3} \dot{\theta}_{w} \\
& -m_{\text {roll }} g c_{w r 2}\left(1+0.06 r_{w}^{2} \dot{\theta}_{w}\right) r_{w}-m_{\text {roll }} g r_{w} \cdot \delta
\end{aligned}
$$

According to the Eqs. 9) - (9) motion equations of disassembled driveline and to the backlash dynamics, the dynamic matrix in backlash mode can be written as:

$\mathbf{A}_{b l}=\left[\begin{array}{llllll}\frac{-b_{\text {total }}}{J_{\text {EngTrDiff }}} & 0 & 0 & 0 & 0 & 0 \\ 0 & \frac{-\frac{1}{2} A_{w} c_{w} \rho r_{w}^{3}-m_{\text {roll }} g c_{w r} 0,0036 r_{w}^{3}}{J_{w}+J_{\text {tire }}+m_{\text {roll }}^{2} r_{w}^{2}} & 0 & 0 & \frac{-m_{\text {roll }} g r_{w}}{J_{w}+J_{\text {tire }}+m_{\text {roll }} r_{w}^{2}} & 0 \\ \frac{1}{i_{\text {tr }} i_{D}} & -1 & 0 & 0 & 0 & 0 \\ -\frac{1}{i_{\text {tr }} i_{D}} & -1 & \frac{k_{\text {drivel }}}{c_{\text {drivel }}} & -\frac{k_{\text {drivel }}}{c_{\text {drivel }}} & 0 & 0 \\ 0 & 0 & 0 & 0 & 0 & 1 \\ 0 & 0 & 0 & 0 & 0 & 0\end{array}\right]$

The nonlinearity appears only in dynamic matrix, input and output matrices remain the same.

$$
\begin{aligned}
& \mathbf{B}_{c o}=\mathbf{B}_{b l}=\left[\begin{array}{llllll}
\frac{1}{J_{E n g T r D i f f}} & 0 & 0 & 0 & 0 & 0 \\
0 & 0 & 0 & 0 & 0 & 1
\end{array}\right]^{T}, \\
& \mathbf{C}_{c o}=\mathbf{C}_{b l}=\left[\begin{array}{llllll}
1 & 0 & 0 & 0 & 0 & 0 \\
0 & 1 & 0 & 0 & 0 & 0 \\
0 & 0 & 0 & 0 & 0 & 0 \\
0 & 0 & 0 & 0 & 0 & 0 \\
0 & 0 & 0 & 0 & 0 & 0 \\
0 & 0 & 0 & 0 & 0 & 0
\end{array}\right]
\end{aligned}
$$

$\left.\begin{array}{llll}-\frac{k_{\text {drivel }} /\left(i_{t r} i_{D}\right)}{J_{\text {EngTrDiff }}} & \frac{k_{\text {drivel }} /\left(i_{t r} i_{D}\right)}{J_{\text {EngTrDiff }}} & 0 & 0 \\ \frac{k_{\text {drivel }}}{J_{w}+J_{\text {tire }}+m_{\text {roll }} r_{w}^{2}} & \frac{-k_{\text {drivel }}}{J_{w}+J_{\text {tire }}+m_{\text {roll }} r_{w}^{2}} & \frac{-m_{\text {roll }} r_{w}}{J_{w}+J_{\text {tire }}+m_{\text {roll }} r_{w}^{2}} & 0 \\ 0 & 0 & 0 & 0 \\ 0 & 0 & 0 & 0 \\ 0 & 0 & 0 & 1 \\ 0 & 0 & 0 & 0\end{array}\right]$

According to its definition a continuous extended Kalman filter does not use the stationary solution of a Riccati equation. It can be formulated using a nonlinear process model for prediction, and its linearization for the on-line calculation of feedback gain. However, since the model only switches between two linear modes and this model treats the phenomena of backlash quite accurately, an extended Kalman filter based on two stationary linear gains are used in this work. In general, the observer can be formulated:

$$
\begin{aligned}
\dot{\hat{\mathbf{x}}} & =\left\{\begin{array}{l}
\mathbf{A}_{\mathbf{c o}} \hat{\mathbf{x}}+\mathbf{B u}+\mathbf{L}_{\mathbf{c o}}(\mathbf{y}-\hat{\mathbf{y}}) \\
\mathbf{A}_{\mathbf{b l}} \hat{\mathbf{x}}+\mathbf{B u}+\mathbf{L}_{\mathbf{b l}}(\mathbf{y}-\hat{\mathbf{y}})
\end{array}\right. \\
\hat{\mathbf{y}} & =\mathbf{C} \hat{\mathbf{x}},
\end{aligned}
$$

where $\mathbf{L}_{c o}$ and $\mathbf{L}_{b l}$ are observer feedback gain matrices designed for their respective cases.

\subsection{Observer realization}

The realization of the observer based on theory and on the simplified two-mass nonlinear driveline model presented above was performed in the following main steps. First the simplified driveline model was parameterized using the parameters of complex driveline model presented in [5]. Then observability of models for both contact and backlash modes were checked. The observer gain was calculated in continuous and discrete time us-

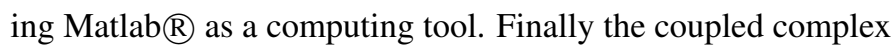
driveline and two-track vehicle models were applied to tuning and optimizing observer performance.

As mentioned before, the model represented in state-space form is only suitable for observer design, if it satisfies observability term. Introducing the backlash model with Eqs. 55 (??) our model dissolved into two parts, the so-called "contact" (co) and "backlash" ( $b l$ modes. The models are linear both in 
contact and backlash modes, and the aim is to find proper observer feedback matrices $\mathbf{L}_{c o}$ and $\mathbf{L}_{b l}$ for both linear models. But for observer design they have to be observable or have to be transformed into observable form. The observability matrix for contact mode has not got full rank, i.e. the model is not observable in state-space form given by matrices $\mathbf{A}_{c o}, \mathbf{B}_{c o}$ and $\mathbf{C}_{c o}$; the number of unobservable states is one.

The non-observable state is the backlash position $x_{4}=\theta_{b}$, which has zero dynamic in contact mode as seen on $\mathbf{a}_{4}$ in Eq. (7); while it is also a non-measured output of the system as shown by the fourth row of output matrix $\mathbf{C}_{c o}$. This statement is really consequent, since the backlash position has to take constant value concerning known backlash size, i.e. $x_{4}=\theta_{b}$ is not to be taken into consideration by the observer in contact mode. For that purpose the unobservable state of the backlash position was eliminated for observer design. The resulted state-space model has minimal order and the same response characteristics as the original model.

Looking at the state-space model of backlash mode, the observability analysis shows two unobservable states. In backlash mode the driveline dissolves actually into two independent rotating parts, to drive and driven moments of inertia as can be seen on Fig. 2. Therefore the torsion $x_{3}=\Delta \theta$ that constitutes originally in contact mode the coherence between the rotating bodies have no physical effect anymore on either moments of inertia, i.e. on the measured outputs of the system. Similarly, as a consequence of the same considerations the backlash position $x_{4}=\theta_{b}$ is the second unobservable state in backlash mode. Since both unobservable states may be computed using observable states $x_{1}$ and $x_{2}$, and a suitable algebraic method based on Eqs. (12) and (13), the estimation of unobservable states is not necessary, i.e. the observer has not to take these into consideration.

$$
\begin{aligned}
& \dot{x}_{3}=\Delta \dot{\theta}=\frac{x_{1}}{i_{\text {tr }} i_{D}}-x_{2} \\
& \dot{x}_{4}=\dot{\theta}_{b}=\frac{x_{1}}{i_{\text {tr }} i_{D}}-x_{2}+\frac{k_{\text {drivel }}}{c_{\text {drivel }}} x_{3}-\frac{k_{\text {drivel }}}{c_{\text {drivel }}} x_{4}
\end{aligned}
$$

The minimal realization of the system is useful for observer design in backlash mode the same way as in contact mode. The resulted feedback matrices were always augmented with zeros for unobservable states in order to get a suitable structure for implementation with state-space models described by matrices $\mathbf{A}_{c o}$, $\mathbf{B}_{c o}$ and $\mathbf{C}_{c o}$, and by matrices $\mathbf{A}_{b l}, \mathbf{B}_{b l}$ and $\mathbf{C}_{b l}$.

\section{Observer evaluation}

The outputs of the observer presented above were also compared with measurement results in order to check the performance of the observer. The measurements were recorded in the vehicle, than the observer was simulated off-line over the measured signals. The torque of the engine, engine speed, wheel speeds and actual gear and gear ratio, each measured on the vehicle CAN, while the driveline torque sensed on the propeller shaft by additional sensor. Twelve $\mathbf{L}$ feedback matrices, twelve
B input matrices and twelve A dynamic matrices for each gear were implemented into the observer both for contact and backlash modes which are shifted, depending on actual gear. However the results presented below were achieved using the same $\mathbf{Q}$ and $\mathbf{R}$ covariance matrices for each gear, some improvement in the observer's performance may probably be achieved by the variation of these.

The results of a tip-in/back-out maneuver demonstrated in Fig. 3 show absolutely the same accuracy that was achieved using the complex model as simulation tool [5].

Looking at the speeds in Fig. 4 the higher accuracy of wheel speed (left) estimated by the observer can be seen compared to the engine speed (middle) that is represented here reduced also to the wheel side of the driveline. This matches expectations according to the actual setup of the design parameter matrices. While the increasing dynamics in the wheel speed are quite well estimated at higher gears too, the accuracy gets worse in the engine speed with rising gear number, but only the amplitudes of the observer's signal miss the measured signal. A limited error in estimated driveline torque appears when backlash is traversing, which is visualized on the right side of Fig. 4

\section{Conclusions}

It was shown in this paper that a simplified two-mass driveline model can be suitable to observer design to estimate driveline torque. The speed measurements on a vehicle can make certain nonlinearities in the driveline visible, usually the effects of clutch and backlash. It was demonstrated that the physical backlash model is applicable not only in backlash size observer [3], but also in the observer for driveline torque estimation. In order to achieve higher accuracy the observer presented here was tuned to follow wheel speed with bigger sensitivity than engine speed, which can be explained with signal qualities in the presented case. Driveline torque control may be a really useful tool to achieve quicker gear-shifting and smaller traction force gap that enhances the longitudinal dynamics. And it can be quite effective to influence additional vehicle body motions. Its benefits can be smaller torque changes during gear-shifting, well damped pitch oscillations that are directly related to longitudinal dynamics. 

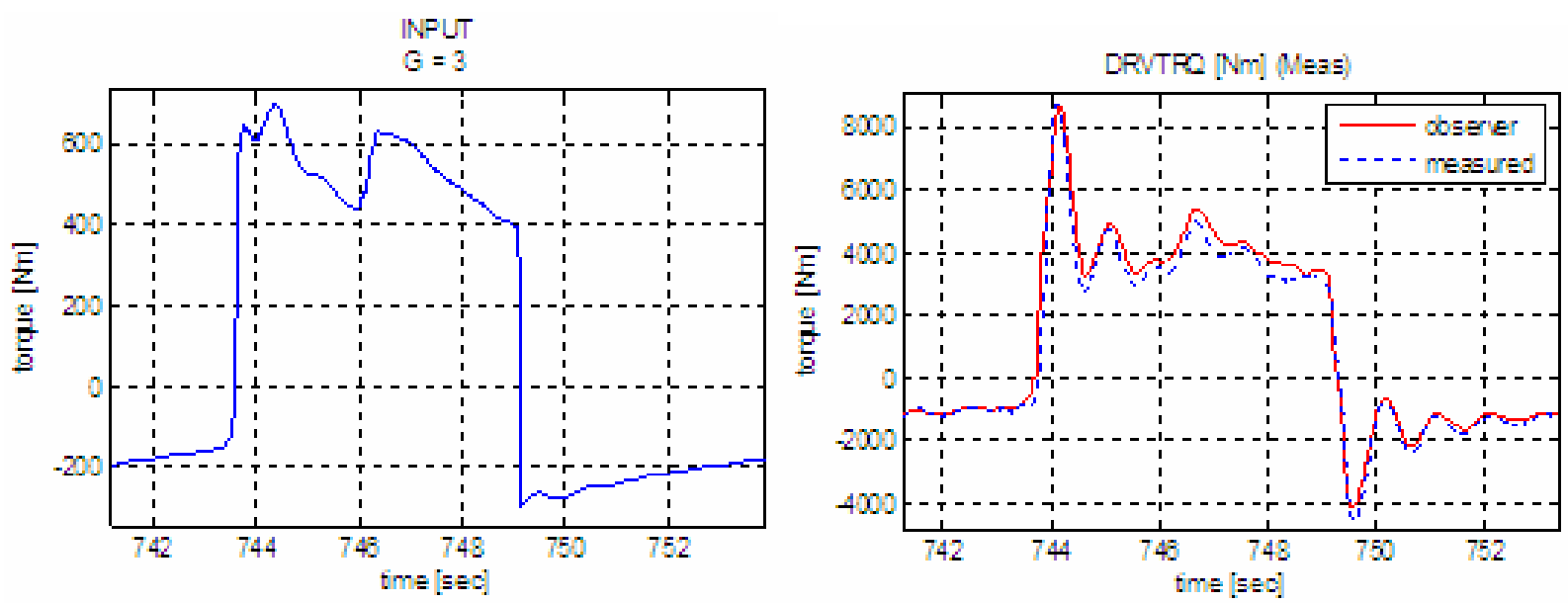

Fig. 3. Observer output driveline torque (right) over system input engine torque (left)
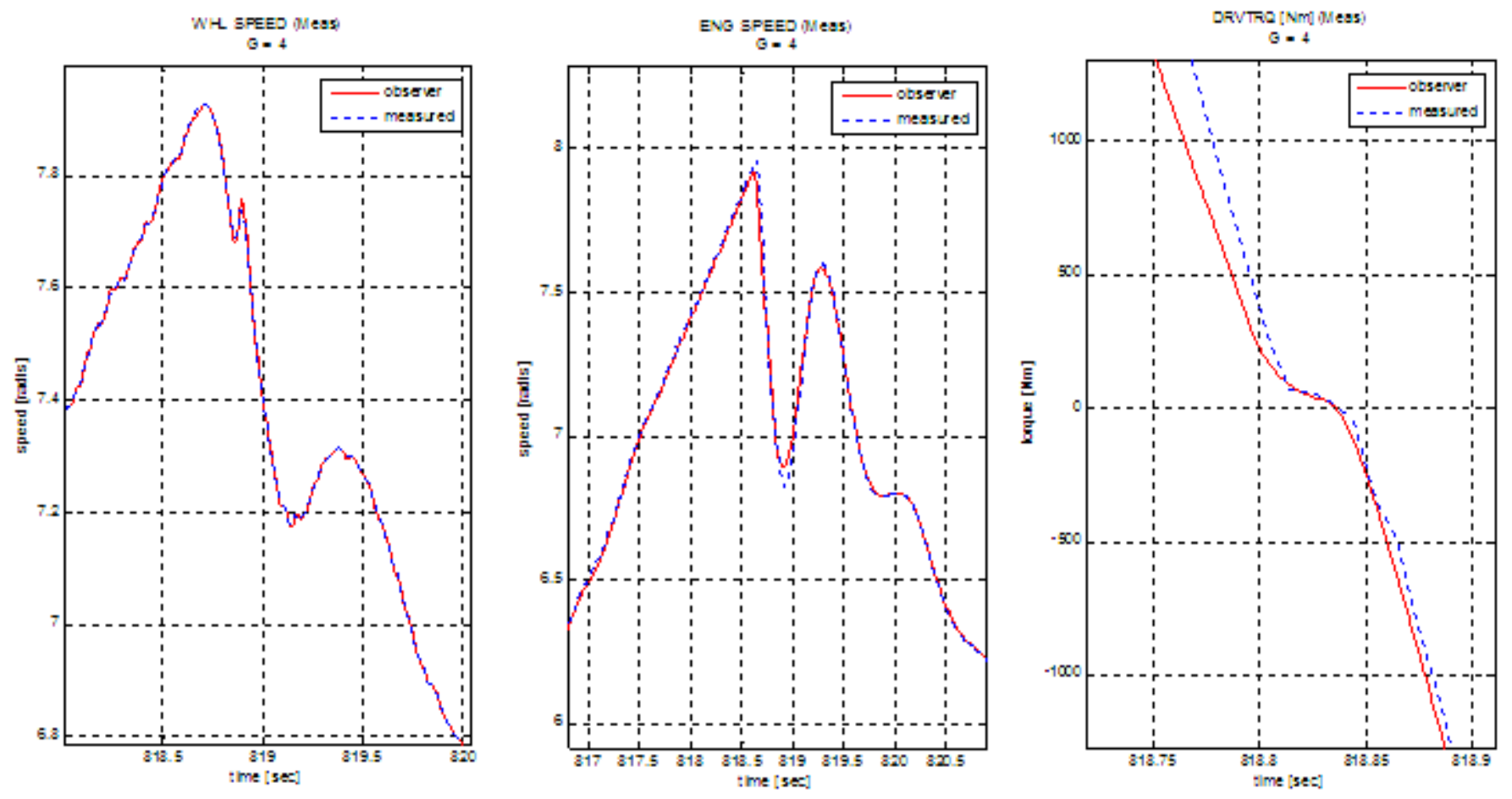

Fig. 4. Measured and observed wheel speed (left), engine speed (middle) and driveline torque (right)

\section{References}

1 Fredriksson J, Egardt B, Nonlinear Control applied to Gearshifting in Automated Manual Transmissions, Proceedings of the 39th IEEE Conference on Decision and Control, (2000).

2 Fredriksson J, Egardt B, Active Engine Control for Gearshifting in Automated Manual Transmissions, International Journal of Vehicle Design, 29((3/4)), (2003), 216-230.

3 Lagerberg A, Egardt B, Estimation of Backlash in Automotive Powertrains, An Experimental Validation, First IFAC Symposium on Advances in Automotive Control, Salerno, Italy, 2004.

4 Nordin M, Galic J, Gutman P O, New Models for Backlash and Gear Play, Int. Journal of Adaptive Control and Signal Processing, 11, (1997), 49-63.

5 Trencséni B, Németh $\mathbf{H}$, Integrated powertrain modelling to enhance comfort of heavy duty vehicles, Proceedings of $22^{\text {nd }}$ JUMV Sience and Motor Vehicles Conference, 2009 